Euskal ikerketen aldizkaria | Revue d'études basques |

Revista de estudios vascos | Basque studies review

2 | 1997

Numéro II

\title{
La situation de la langue basque en Pays Basque Nord
}

\section{Bernard Oyharçabal}

\section{OpenEdition}

\section{Journals}

Édition électronique

URL : http://journals.openedition.org/lapurdum/1777

DOI : 10.4000/lapurdum. 1777

ISSN : 1965-0655

\section{Éditeur}

IKER

Édition imprimée

Date de publication : 1 octobre 1997

Pagination : $29-43$

ISBN : 2-84127-142-0

ISSN : $1273-3830$

Référence électronique

Bernard Oyharçabal, «La situation de la langue basque en Pays Basque Nord », Lapurdum [En ligne], 2 | 1997, mis en ligne le 01 septembre 2010, consulté le 30 janvier 2020. URL : http:// journals.openedition.org/lapurdum/1777 ; DOI : 10.4000/lapurdum.1777 


\section{B. OYHARÇABAL}

\section{LA SITUATION DE LA LANGUE BASQUE EN PAYS BASQUE NORD ${ }^{1}$}

Lorsque durant le Premier Empire Coquebert de Montbret en charge du bureau de Ia statistique au ministère de l'Intérieur, adresse aux préfets de l'Empire son questionnaire relatif aux parlers utilisés dans leur circonscription, le préfet Castellane en place dans les Basses-Pyrénées en 1806, traça sur une carte du département une ligne marquant les limites de la zone bascophone (document reproduit dans Oyharçabal 1992), et dressa la liste des villages limitrophes en accompagnant ce relevé de quelques brefs commentaires relatifs à certaine localités qu'il considérait comme bilingues (Brunot, 1927).

$S^{`}$ agissant, selon tous les témoignages de l'époque, d'une zone linguistiquement homogène, $c^{\circ}$ est sur cette base que, pour la première fois, furent dénombrés les bascophones du Pays Basque aquitain, c'est-à-dire par simple addition du nombre d'habitants des diverses localités incluses dans la zone bascophone. Le dénombrement publié dans l'annuaire du Bureau des Longitudes présenté en 1809 fut de 108000 locuteurs bascophones.

Hélas. certe attention portée par l'administration française aux faits de langue de cette nature n'eut guère de prolongement, et jamais depuis lors aucun effort n'a été fait pour dénombrer les locuteurs bascophones, et encore moins bien sûr pour affiner cette connaissance. Les chiffres rapportés un peu plus tard, en particulier durant la seconde partie du 19ème siècle par F. Michel (135 000), E. Reclus (120000 au maximum), et L-L. Bonaparte (125000), suivi par J. Vinson, semblent avoir été fondés sur la même démarche que celle de 1806: Il en fut de même au $20^{\circ}$ siècle. Les estimations ainsi obtenues avaient une certaine fiabilité, car jusqu'à la période d'après-guerre le Pays Basque aquitain a conservé une relative stabilité en ce qui concerne la bascophonie. Il était possible dans ces conditions d'évaluer approximativement le nombre des habitants sachant le basque dans la zone bascophone à partir du nombre d'habitants. C'est encore sur cette base qu'à la fin des années 1960 Yrizar (1973) fonda son enquête, en dénombrant un total de 85000 locuteurs environ; il dut toutefois, pour obtenir ce nombre, pondérer les chiffres de population en demandant aux responsables civils ou religieux en charge sur place de lui foumir une estimation en pourcentage des bascophones dans les communes.

C'était là toutefois un pis-aller, qui au surplus devint bientôt pratiquement impossible à utiliser, même dans le Pays Basque à dominante rurale. Au demeurant celui-ci était sur le recul, représentant un pourcentage des habitants de plus en plus faible, et connaissant un déplacement de sa population vers les zones plus urbanisées de la côte et notamment de l'agglomération bayonnaise. Par ailleurs, il était difficile de continuer à ignorer celle-ci, exclue de la zone bascophone traditionnelle et où se trouvait concentrée non loin de la moitié de la population du Pays Basque, et vers laquelle se dirigeait une partie non négligeable des personnes quittant le Pays Basque intérieur. 
En ce qui concerne les études de démographie linguistique, la situation du Pays Basque ibérique ne fut pas meilleure jusqu'à une date récente. Cependant, au début des années 1980, peu après la fin du franquisme, la reconnaissance du basque comme langue officielle en Navarre et dans les provinces de la Communauté Autonome du Pays Basque, et la mise en place d'une nouvelle administration régionale en Espagne, les recensements inclurent des questions relatives à la connaissance des langues officielles d'Espagne, ce qui permit d'effectuer dans la Communauté Autonome les premiers travaux de démolinguistique du Pays Basque, lesquels furent basés sur le recensement de 1986 (HPIN 1989).

C'est après cette première étude qu'il fut décidé d'entreprendre en 1991 une véritable enquête sociolinguistique, non plus dans la seule Communauté Autonome, mais sur l'ensemble du Pays Basque, dans la mesure où institutionnellement cela s'avérait possible. Cette enquête fut effectuée dans de bonnes conditions dans la Communauté Autonome, et également en Navarre, dont le Gouvernement provincial s'associa au projet. En Aquitaine, en l'absence d'instances administratives se consacrant à la politique linguistique au sein des institutions départementales ou régionales, l'Institut Culturel Basque fut sollicité, mais de manière très tardive car lui-même de création récente. C'est donc en urgence, et dans des conditions pas vraiment satisfaisantes sur le plan technique, que l'enquête fut réalisée au nord de la frontière. Il convient toutefois de dire que cette première expérience, malgré des circonstances difficiles, permit de procéder à une première approche quantitative et d'élaborer des analyses comparatives fort utiles (Aizpurua, 1995) ${ }^{3}$.

En 1995, Jorsque le gouvernement de la Communauté Autonome et le Gouvernement de Navarre convinrent de réaliser une nouvelle enquête l'année suivante, cinq ans après celle de 1991, le Pays Basque aquitain fut dès le départ associé à l'élaboration de l'enquête. Un comité de pilotage fut mis en place par l'Institut Culturel Basque avec la participation de linguistes et de sociologues. L'INSEE fut sollicité pour élaborer des échantillons destinés à la réalisation des enquêtes. A défaut d'un support financier de la part des autorités publiques nationales ou locales, c'est auprès des institutions de l'Union Européenne que fut obtenue la plus grande partie du financement de l'enquête en Pays Basque aquitain.

La présente communication expose et analyse les principaux résultats relatifs à la compétence en basque de la populatjon de plus de 16 ans résidant dans le Pays Basque aquitain, tels qu'ils apparaissent à la suite de l'enquête de $1996^{4}$.

\section{L'enquête de 1996}

L'enquête de 1996 a été réalisée par la méthode des sondages sur l'ensemble du Pays Basque, mais par des organismes spécifiques selon les parties du Pays Basque concernéess. L'ensemble des résultats ont été regroupés et traités par informatique à Vitoria-Gasteiz sous la responsabilité du cabinet technique du secrétariat à la politique linguistique du Gouvernement de la Communauté Autonome du Pays Basque.

\subsection{Les zones d'enquêtes}

Comme en Pays Basque Sud, où les zones non bascophones ou débasquisées, parfois depuis des siècles, sont inclues dans les enquêtes linguistiques, et comme ce fut le cas en 1991. lenquête de 1996 a été effectuée pour la partie aquitaine avec pour base la population de 16 ans et plus, résidant dans la partie du. département des PyrénéesAtlantiques qui correspond au Pays Basque de France tel que celui-ci est délimité 
aujourd hui dans diverses instances d'études ou de réflexion mises en place par l'administration préfectorale, départementale et consulaire, et qui suit les limites du département y compris les zones situees sur la rive droite de l'Adour.

Compte tenu d'une part de l'hétérogénéité sociologique et démographique qu'on y observe, et d'autre part de l'inégale répartition des bascophones, ce territoire a été divisé dans le cadre de l'enquête en quatre zones correspondant à une partition sociodémographique recoupant partiellement les limites traditionnelles linguistiques et historiques. Les quatre zones sont les suivantes :

L'agglomération Biarritz-Anglet-Bayonne (avec le canton du Boucau), qui est très urbanisée, et qui n'appartient pas à la zone bascophone traditionnelle ${ }^{6}$.

La côte-sud, qui englobe les cantons incluant les villes de la bordure maritime au sud de Bidart jusqu'à la frontière ; elle correspond à deux cantons du Labourd appartenant à la zone bascophone traditionnelle et centrés sur deux villes côtières : Hendaye et Saint-Jean-de-Luz.

Le Labourd intérieur, qui englobe les cantons labourdins qui n' incluent pas de commune côtière : il s'agit - en dehors de quelques communes de la bordure de l'Adour d'une zone traditionnellement bascophone, appartenant jusqu'à une date récente au Pays Basque rural, mais qui abrite désormais une population dont une proportion non négligeable, voire importante ou très majoritaire, travaille dans l'agglomération bayonnaise ou dans les villes de la côte. Sur le plan démographique, ces cantons se caractérisent par rapport aux autres cantons du Pays Basque intérieur par le fait qu'ils ont présenté un solde démographique positif entre les deux derniers recensements.

La Basse-Navarre et la Soule. Traditionnellement bascophone, cette zone, qui correspond aux anciennes provinces intérieures du Pays Basque aquitain, regroupe dans l'enquête les cantons à solde démographique négatif.

Pour chacune de ces zones, des échantillons de la population ayant 16 ans d'âge ou plus, ont été définis, et fournis à partir des listes des annuaires téléphoniques, sans tenir compte de l'origine, ni de la nature de la résidence. Les enquêtes ont eu lieu en février-mars 1996 auprès de 1400 personnes.

Les résultats ont été ensuite pondérés selon la distribution de la population sur la base du recensement de 1990 et sa projection en fonction du sexe et de l'âge.

\subsection{Le questionnaire}

Un questionnaire unique a été élaboré pour l'ensemble des enquêtes qu'elle fussent réalisées dans la Communauté Autonome, en Navarre, ou en Pays Basque aquitain, avec quelques retouches d'adaptation lorsque cela s'avérait nécessaire. Ceci permet un traitement unique des données recueillies.

Le questionnaire comprend une cinquantaine de questions que l'on peut regrouper. outre celles relatives à la personne interrogée, en trois gros chapitres : questions concernant la compétence en basque, questions concemant le comportement (emploi effectif du basque), questions concernant les opinions ou les attitudes à l'égard du basque.

\subsection{Les types de locuteurs}

Dans une situation telle que celle de la langue basque, l'une des difficultés rencontrées pour quantifier les locuteurs réside dans la d̦éfinition des types de compétence permettant de prendre en compte les situations intermédiaires. 
Le questionnaire a visé à définir les types de locuteurs sur la seule compétence en basque et en français en Pays Basque Nord ou en espagnol en Pays Basque Sud. La typologie suivante a été élaborée pour la partie aquitaine ${ }^{8}$ :

- bascophones, non francophones ou francophones passifs ;

$\begin{array}{ll}\text { - bilingues } & \text { - avec basque dominant } \\ & \text { - équilibrés } \\ \text { - avec français dominant }\end{array}$

- francophones, bascophones passifs ;

- francophones non bascophones.

Pour des raisons de clarté et de simplicité, nous ne retiendrons pas compte dans notre présentation que trois types de locuteurs:

Les bascophones à compétence active (bascophones actifs dans les tableaux). Nous ne distinguerons pas bilingues francophones et non-francophones, la proportion de bascophones ignorant le français étant statistiquement négligeable, puisqu'elle est inférieure à $1 \%$. De même nous rassemblons dans cette même catégorie les différents types de bilingues, pour ne retenir comme critere que le fait d'avoir ou non une compétence active en basque. Indiquons simplement que les trois types de bilingues sont en proportion sensiblement égale.

Les bascophones à compétence passive (bascophones passifs dans les tableaux). Il s'agit d'une catégorie intermédiaire sans doute quelque peu hétérogène ; elle regroupe les personnes indiquant pouvoir parler un peu en basque, ou être en mesure de bien ou assez bien le comprendre, à l'écrit ou à l'oral.

Les non-bascophones. Cet ensemble regroupe les personnes disant ignorer totalement le basque, ou n'en connaissant que quelques mots ou ne le comprenant qu' un peu.

Les options offertes dans les réponses pour auto-évaluer la compétence était pour les quatre types de compétence (compréhension orale, expression orale, compréhension de l'écrit, expression écrite) : rien, quelques mots, un peu, assez bien, bien.

\subsection{L'origine et la langue maternelle de la population interrogée}

Il convient de signaler également certaines caractéristiques générales concernant l'échantillon ou bien la population et pesant directement sur la compétence linguistique des personnes.

- Le premier élément concerne le nombre de bascophones dans l'échantillon, avant que ne soient effectuées les répartitions : 480 personnes (soit $34 \%$ ) étaient bascophones et 920 non-bascophones $(66 \%)^{9}$.

- Le second élément est relatif au lieu de naissance : sur l'ensemble des zones d'enquête les non- natifs représentent $38 \%$; entre $16 \%$ dans la zone 3 (BasseNavarre et Soule) et $51 \%$ dans la zone 1 correspondant à l'agglomération bayonnaise. Ces chiffres sont évidemment très importants : en effet dans la situation sociolinguistique qui est celle du basque, où le seul mode d'acquisition du basque a été la transmission familiale, il ne peut s'agir statistiquement que d'une population non bascophone.

- Le troisième élément regarde le lieu de résidence. Le tableau 1.A. ci-dessous indique quelle est, selon le recensement de 1990, la population de 16 ans et plus de chacune des zones d'enquêtes et le nombre d'enquêtes réalisées dans chacune d'elles. 
TABLEAU I.A. Population de 16 ans cl plus en chiffres absolus (recensement de 1990) ct nombres d'enquêtes réaliscées en 1996 dans chaque zone

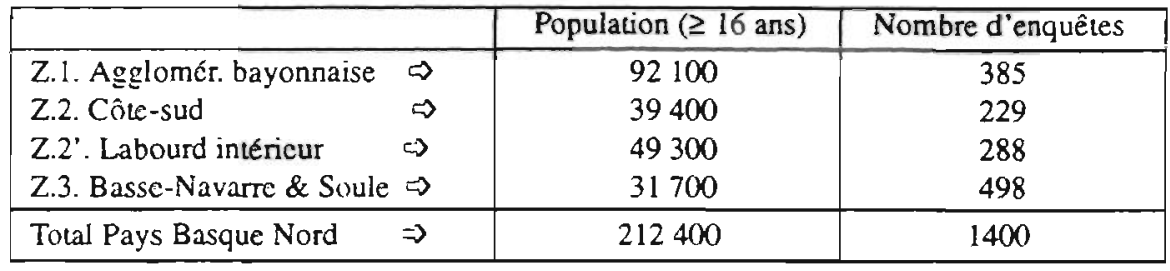

Comme on peut le constater le nombre d'enquêtes réalisées dans les zones 2 et 2 'est faible et leur regroupement est souhaitable, et même nécessaire pour une exploitation plus fine des résultats ${ }^{10}$.

\section{Résultats généraux relatifs à la compétence}

Les tableaux 2.A et 2.B présentent les résultats en nombres absolus et en pourcentage regardant la population bascophone et non-bascophone.

TABLEAU 2.A. Nombre de basconhones $\mathrm{cl}$ non-bascophones au scin de la population de 16 ans $\mathrm{ct}$ plus du Pays Basque aquitain. et répartition dans les différentes zones d'enquête

\begin{tabular}{|l|c|c|c|c|c|}
\hline & Total & Z.I. & Z.2 & Z.2 & Z.3 \\
\hline bascophones actifs $\Rightarrow$ & 56200 & 8400 & 8100 & 19400 & 20300 \\
bascophones passifs $\Rightarrow$ & 19800 & 3600 & 6000 & 6300 & 3900 \\
non bascophoncs $\Rightarrow$ & 136400 & 80100 & 25200 & 23600 & 7500 \\
\hline
\end{tabular}

TABLEAU 2.B. Pourcentage des bascophones er non-bascophones au sein de la population de 16 ans el plus du Pays Basque aquitain et des 4 zones d'enquéte

\begin{tabular}{|c|c|c|c|c|c|}
\hline & Total & Z.I. & $\mathrm{Z} .2$ & Z.2' & Z.3 \\
\hline bascophones actifs $\Rightarrow$ & 26.43 & 9.08 & 20.76 & 39,24 & 63,99 \\
\hline bascophoncs passirs $\rightarrow$ & 9,34 & 3.90 & 15.30 & 12.85 & 12.27 \\
\hline non bascophoncs $\Rightarrow$ & 64.23 & 87.02 & 63.94 & 47.90 & 23.74 \\
\hline TOTAL & 100.00 & $100 .(x)$ & 100.00 & 99.99 & 100.00 \\
\hline
\end{tabular}

Comme le montrent ces tableaux, la proportion des personnes résidant en Pays Basque aquitain et ayant une compétence active en basque correspond à un gros quart de la population. Celle de ceux n'ayant qu'une connaissance passive du basque approche les $10 \%$. Près de deux sur trois ignorent le basque.

Le nombre de personnes ayant 16 ans ou plus en mesure de parler le basque est d'environ 56000 personnes sur une population totale de plus de 16 ans évaluée à 212 000. Le nombre des bascophones passifs est approximativement de 20000 .

Une nette majorité des bascophones actifs vit dans les cantons non côtiers (zones 2 'et 3). où ils sont 39700 . soit $70 \%$ du total.

Néanmoins $15 \%$ des bascophones vivent dans l'agglomération bayonnaise ( 8200 personnes). 
Si l'on tient compte de la répartition géographique, le nombre de bascophones ayant une compétence active n'est nettement majoritaire (près de 2 habitants sur 3) que dans la zone 3, correspondant à la Basse-Navarre et à la Soule, région économiquement et démographiquement en difficulté, puisqu'elle est composée des cantons ayant présenté un solde démographique négatif entre les précédents recensements.

La différence entre l'agglomération bayonnaise et le reste de la côte de Bidart à Hendaye est sensible : en effet l'agglomération bayonnaise, qui n'appartient pas à la zone traditionnellement bascophone, a une proportion de bascophones actifs inférieure à $10 \%$, contre $20 \%$ pour le reste de la côte. Dans le Labourd intérieur cette proportion est nettement supérieure : $40 \%$.

La répartition par zones est manifestement pertinente, comme le montre l'approximation du nombre de bascophones pour 10 habitants dans chacune des zones telle qu'elle est résumée dans le tableau 2.C :

TABLEAU 2.C. Approximation du nombre de bascophones pour 10 habitants sclon les zones

\begin{tabular}{|lll|}
\hline Z. 1. Agglomération bayonnaise & $\Rightarrow$ & $1 / 10$ habitants \\
Z. 2. Labourd-Sud côtier & $\Rightarrow$ & $2 / 10$ habitants \\
Z. 2'. Labourd intérieur & $\Rightarrow$ & $4 / 10$ habitants \\
Z 3. Soule \& Basse-Navarre & $\Rightarrow$ & $6 / 10$ habitants
\end{tabular}

Il peut être intéressant de comparer ces résultats avec les travaux démolinguistiques effectués auparavant et mentionnés en introduction (Coquebert de Montbret 1806, Reclus 1867. Yrizar 1973). Comme ces derniers se limitaient à la prise en considération de la seule zone bascophone traditionnelle, il convient de retrouver une base de comparaison territoriale identique. La partition en zones utilisée permet d'effectuer une approche assez fine, en excluant l'agglomération bayonnaise (zone l) du territoire d'enquête. En effet, même si les communes de la bordure de l'Adour n'appartenant pas à la zone bascophone traditionnelle (Urt, La Bastide-de-Clairence, Bidache) sont inclues dans la zone 2 du fait des découpages cantonaux, l’incidence statistique de cette inclusion peut être considérée comme réduite.

TABLEAU 2.D. Nombre et pourcentage des bascophones et non bascophones de 16 ans et plus dans la zone hascophone traditionnclle

\begin{tabular}{|c|c|c|c|}
\hline & & Nombrc & Pourcentage \\
\hline bascophones actirs & $\rightarrow$ & 47800 & $40 \%$ \\
\hline bascophones passils & C) & 16200 & $13 \%$ \\
\hline non bascophones & $\rightarrow$ & 56300 & $47 \%$ \\
\hline TOTAL & $\Rightarrow$ & 120300 & $100 \%$ \\
\hline
\end{tabular}

Le tableau 2.D foumit l'évaluation en nombre et pourcentage des bascophones et non-bascophones dans la zone bascophone traditionnelle (zones $2,2^{\prime} \mathrm{et} 3$ ). Comme on le voit. si la présence du basque est mieux marquée que par rapport à l'ensemble Pays Basque aquitain, près de la moitié de la population $(47 \%)$ de la population ignore le basque. Rappelons que lors des enquêtes du 19ème siècle ce territoire était considéré comme universellement bascophone en dehors des personnes récemment installées 
dans les villes. La population n'ayant guère varié depuis le recensement de 1866 sur cette zone ( 120300 selon le recensement de 1990 pour les plus de 16 ans, 123000 pour la population totale en $1866 \mathrm{~d}$ après E. Reclus), on peut considérer que la perte du basque sur cette zone a été d'environ $60 \%$.

\section{Transmission familiale et acquisition tardive ou perte de compétence en basque}

L'enquête met en évidence que pour la population considérée la transmission familiale est le moyen d'acquisition pratiquement unique de la langue basque.

Ceci n'est pas surprenant étant donné l'éviction du basque du système d'enseignement jusqu'à une date récente, et son exclusion de fait de la vie sociale publique.

Pour mesurer ceci on peut d'abord se reporter au tableau suivant (3.A) qui fait apparaître la compétence en basque selon que l'euskara est ou non langue matemelle.

TABLEAU 3.A. Types de compćtence en basque sclon la langue matemelle (évaluations en pourcentage sur la population totale)

\begin{tabular}{|l|c|c|c|c|c|}
\hline & $\begin{array}{c}\text { Personnes dont } \\
\text { le basque cst la ou } \\
\text { unc des langue(s) } \\
\text { matemelle(s) }\end{array}$ & $\begin{array}{c}\text { Pcrsonnes dont } \\
\text { le basque n'est pas } \\
\text { la ou l'une des } \\
\text { langue(s) } \\
\text { matemelle(s) }\end{array}$ & $\begin{array}{c}\text { Population } \\
(216 \text { ans })\end{array}$ \\
\hline paricnt bicn le basquc \\
partent un peu le basque \\
ne parleni pas lc hasque
\end{tabular}

Comme on le voit, $1 \%$ seulement de la population totale est constitué de personnes n’ayant pas le basque comme langue maternelle mais ayant une compétence active. Le nombre pour la compétence passive n'est guère supérieur $(3,5 \%)$. En un mot, très peu de personnes n'ayant pas eu le basque comme langue maternelle acquièrent la capacité d'utiliser sans difficultés cette langue.

Le basque est la langue matemelle d'un petit tiers des habitants, et c'est presque exclusivement parmi eux que se comptent les bascophones actifs.

Le même tableau (3.A) fait également apparaître la perte d'une compétence active en basque chez les personnes ayant eu le basque comme langue maternelle. Cette perte est certes limitée, mais néanmoins sensible puisqu'elle correspond à $6 \%$ de la population totale, soit environ $20 \%$ de la population ayant eu le basque comme langue maternelle.

Contrairement à ce que prétend l'idée reçue, le basque appris dans l'enfance se perd s il n'est pas employé, et cette circonstance se trouve réalisée aujourd'hui en Pays Basque même.

La difficulté d acquérir le basque hors de la famille est également mise en évidence lorsque l'on considère la compétence en basque des groupes de population constitués 
selon le lieu de naissance. Quatre groupes ont été formés selon que les personnes étaient nées ou non en Pays Basque, et également, lorsque tel était le cas, selon que les deux parents ou l'un des deux étaient eux-mêmes natifs. Les résultats sont ceux du tableau suivant (3.B).

TABLEAU 3.B. Mcsurc de l'acquisition par immersion sociale: pourcentage des bascophones ct non-bascophones dans la population classé selon l'origine géographique des personnes et celle de leur parcnts (critère du licu de naissance)

\begin{tabular}{|l|c|c|c|c|}
\hline & $\begin{array}{c}\text { bascophones } \\
\text { actifs }\end{array}$ & $\begin{array}{c}\text { bascophoncs } \\
\text { passifs }\end{array}$ & $\begin{array}{c}\text { non } \\
\text { bascophones }\end{array}$ & Total \\
\hline natif \& 2 parents natifs & 53 & 15 & 33 & 100 \\
nauif \& 1 parcnt natif & 9 & 7 & 83 & 100 \\
natif \& parcnts non-natifs & 5 & 5 & 90 & 100 \\
non-natifs & 4 & 4 & 92 & 100 \\
\hline TOTAL & 26 & 9 & 64 & 100 \\
\hline
\end{tabular}

Le tableau ci-dessus (3.B) confurme qu'en raison du caractère exclusif de la transmission familiale comme facteur de maintien transgénérationnel de la langue, l'origine géographique des personnes est un élément déterminant au regard de la compétence linguistique en basque.

En effet un très faible pourcentage parmi les non-natifs, ou les enfants de nonnatifs, déclare avoir une compétence active. La basquisation par immersion des personnes non natives est certes bien attestée jusqu'à une date relativement récente dans le Pays Basque rural, mais cette immigration est très minoritaire, et l'acquisition tardive par immersion a probablement cessé de jouer à peu près partout depuis l'aprèsguerre.

La proportion de non-bascophones chez les non-natifs d"une part, et les natifs ayant les deux parents. ou a fortiori un seul des parents, non natifs, d'autre part, ne varie pas considérablement ( 8 ou 9 sur 10). Autrement dit il n'y a pratiquement pas d'acquisition de la compétence en basque à la deuxième génération.

Nous ne possédons la ventilation par zone et par âge de ces chiffres (au demeurant ils seraient difficilement exploitables en raison de l'étroitesse de l'échantillon), mais il est probable que les personnes elles-mêmes non natives ou ayant un des parents non natif et étant bascophones ( 1 à 2 sur 10 respectivement pour chacun des deux groupes) correspondent à des personnes à la fois âgées et vivant en Pays Basque intérieur, c'est-à-dire ayant connu une situation où la présence sociale du basque permettait l'apprentissage par immersion dans la vie locale. Il est difficile d'estimer ici la part des personnes ayant appris le basque à la suite d'une démarche personnelle volontaire, car le dénombrement de ces bascophones n'apparaît pas dans l'enquête. Néanmoins il serait surprenant que ce nombre soit statistiquement significatif. Ces éléments sont très importants puisqu'ils montrent clairement que, contrairement à ce qu'il en a été dans un passé peu lointain dans la zone bascophone traditionnelle, il n'y a plus actuellement d'intégration linguistique des populations non-natives, lesquelles représentent pourtant un pourcentage important de la population totale $(38 \%)$.

Le tableau suivant (3.C) fournit un autre élément permettant d'apprécier l'importance de la transmission familiale. Il fait apparaitre le pourcentage de bascophones de langue maternelle en fonction de la compétence en basque des parents. 
TABLEAU 3.C. Transmission du basque comme langue maternclle selon la compétence linguistique des parents en basque (pourcentages)

\begin{tabular}{|c|c|c|}
\hline & $\begin{array}{c}\text { locuteurs avec } \\
\text { langue(s) } \\
\text { matemelle(s) } \\
\text { incluant le basque }\end{array}$ & $\begin{array}{c}\text { locuteurs avec } \\
\text { langue(s) } \\
\text { maternelle(s) } \\
\text { n'incluant pas } \\
\text { le basque }\end{array}$ \\
\hline $\begin{array}{l}\text { Père ct mère bascophones }(100) \\
\text { père bascophone, } \\
\text { Mère non bascophone }(100) \\
\text { mère bascophone. } \\
\text { Pc̀re non bascophone }(100) \\
\text { Père et mère } \\
\text { non-bascophones }(100)\end{array}$ & $\begin{array}{l}83 \% \\
15 \% \\
26 \% \\
00 \%\end{array}$ & $\begin{array}{l}17 \% \\
85 \% \\
74 \% \\
100 \%\end{array}$ \\
\hline
\end{tabular}

Le tableau ci-dessus (3.C) montre que parmi les personnes interrogées dont le père et la mère étaient bascophones $17 \%$ n'ont pas eu le basque comme première langue (en même temps éventuellement que le français), ce qui représente la mesure de la rupture de la transmission familiale. On observera également que selon ce tableau l'acquisition par apprentissage tardif chez les enfants de non-bascophones n'est pas statistiquement observable.

Cette rupture est évidemment beaucoup plus marquée lorsqu'un des deux parents n'est pas bascophone. Soulignons qu'alors l'écart observé n'est pas proportionnel, mais qu'il est considérable, en particulier s'il s'agit de la mère : en effet, en ce cas la rupture dans la transmission est de $85 \%$.

Cette différence confirme, au niveau de la famille, le principe bien connu concernant les langues minorées dans les ensembles à bilinguisme partiel (c est-à-dire une situation où seuls les locuteurs d'une langue minorée ont une double compétence), et selon lequel la place prise par la langue minorée dans les choix linguistiques des locuteurs n'est en rien proportionnelle au nombre de locuteurs de cette langue au sein du groupe.

\section{Compétence en basque selon les générations}

Les données les plus inquiétantes apportées par l'enquête sont sans doute celles qui concernent la répartition par classes d'âge des résultats relatifs à la compétence.

TABLEAU 4.A. Répartition en pourcentages de la population (non-) bascophone sclon lcs classcs d'äge en Pays Basque aquitain (population $\geq 16$ ans)

\begin{tabular}{|ll|c|c|c|c|}
\hline & & $\begin{array}{c}\text { bascophones } \\
\text { actifs }\end{array}$ & $\begin{array}{c}\text { bascophones } \\
\text { passifs }\end{array}$ & $\begin{array}{c}\text { non } \\
\text { bascophones }\end{array}$ & Total \\
\hline $16-24$ ans & $\Rightarrow$ & 11.5 & 13.00 & 75.5 & 100 \\
$25-34$ ans & $\Rightarrow$ & 13.50 & 13.5 & 73.00 & 100 \\
$35-49$ ans & $\Rightarrow$ & 27.5 & 8.5 & 64.00 & 100 \\
$50-64$ ans & $\Rightarrow$ & 31.00 & 9.00 & 60.00 & 100 \\
$\geq 65$ ans & $\Rightarrow$ & 37.5 & 6.00 & 56.5 & 100 \\
\hline
\end{tabular}


Comme on le voit, la proportion de bascophones au sein de la population baisse très significativement avec l'âge. De plus d'un tiers chez les plus de 65 ans $(37,5 \%)$, cette proportion descend à $11,5 \%$ chez les 16-24 ans.

La plus grosse rupture, avec une chute de moitié dans cette proportion se situe dans la génération née après la guerre : en effet, si dans la génération née approximativement entre 1946 et 1960 les bascophones représentent $27,5 \%$, pour celle née entre 1961 et 1970 ce rapport descend à $13,5 \%$.

Pour la génération née après 1970 la baisse se confirme, mais avec un ralentissement sensible dans le rythme.

Ces données (tableau 4.A) contrastent fortement avec les résultats observés en Pays Basque Sud, où c'est la tranche d'âge la plus jeune qui montre la plus forte proportion de bilingues actifs, dépassant même celle observée chez les plus de 65 ans. Le tableau suivant (4.B) montre quels sont les résultats de l'enquête dans la Communauté Autonome du Pays Basque :

TABLEAU 4.B. Répartition en pourcentages de la population (non-) bascophone

sclon les classes d'âge dans la Communauté autonome du Pays Basque (population $\geq 16$ ans)

\begin{tabular}{|ll|c|c|c|c|}
\hline & & $\begin{array}{c}\text { bascophones } \\
\text { actifs }\end{array}$ & $\begin{array}{c}\text { bascophoncs } \\
\text { passifs }\end{array}$ & $\begin{array}{c}\text { non } \\
\text { bascophones }\end{array}$ & Tolal \\
\hline $16-24$ ans & $\Rightarrow$ & 33 & 37 & 30 & 100 \\
$25-34$ ans & $\Rightarrow$ & 25 & 27 & 48 & 100 \\
$35-49$ ans & $\Rightarrow$ & 21 & 11 & 68 & 100 \\
$50-64$ ans & $\Rightarrow$ & 21 & 6 & 73 & 100 \\
$\geq 65$ ans & $\Rightarrow$ & 26 & 5 & 67 & 98 \\
\hline
\end{tabular}

Le tableau ci-dessus (4.B) est intéressant car il montre les effets relativement rapides d'une action volontariste en faveur du bilinguisme afin d'accroître le nombre de locuteurs compétents dans une langue historiquement minorée devenue nettement minoritaire.

Le contraste avec les résultats du Pays Basque aquitain est frappant. Pourtant celui-ci partail après la guerre d'une situation démolinguistique bien meilleure, et les circonstances politiques ne furent guère favorable au basque pendant longtemps outreBidassoa.

C'est en effet avec la génération née après 1945 que cesse dans les provinces de la Communauté Autonome la chute du pourcentage des bascophones au sein de la population de chaque classe d'âge. Avec les générations nées après 1960 commence le processus de remontée qui s̀accélère ensuite avec les générations nées après 1970.

Tandis que la génération des 25-50 ans, née entre 1945 et 1970, a accéléré considérablement le processus de débasquisation en Pays Basque aquitain, au contraire, dans la Communauté Autonome, elle est celle qui a fait cesser, puis inversé, ce processus.

\section{Conclusion}

Nous nous sommes limités dans cette présentation aux données relatives à la compétence. sans nous arrêter sur d'autres facteurs intervenant également dans l'examen des situations linguistiques : à savoir les circonstances et conditions d'emploi effectif 
de la langue, et, dans une moindre mesure, les opinions et les attitudes. Mais ces éléments suffisent amplement pour formuler un diagnostic hélas sans équivoque : la langue basque est dans une siruation critique sur le territoire français, et sa survie sociale gravement en cause.

La transmission familiale, qui s'est pourtant maintenue à une niveau relativement élevé jusqu'à une date récente, et qui s'avère plus que jamais indispensable à la continuité de la langue, n'est plus en mesure de préserver à elle seule la présence sociale du basque pour l'avenir. La chute dramatique de la proportion des bascophones actifs dans les classes d'âge les plus jeunes ne laisse aucune espèce de doute sur ce point.

Les facteurs pesant sur l'évolution de la géographie (concentration urbaine, dévitalisation du Pays Basque intérieur demeuré socialement le plus bascophone) et des flux migratoires (importance grandissante de la proportion des personnes nées hors du Pays Basque et qui se trouvent privées, de fait, de toute possibilité d'intégration ou d'accommodation linguistique respectueuse du basque) rendent donc indispensable la mise en place d'une politique linguistique spécifique en faveur de la langue basque, dans l'enseignement bien sûr, mais également dans l'organisation quotidienne de la vie sociale. y compris dans les zones les plus urbanisées, démographiquement dynamiques et porteuses des modèles de comportement les plus prégnants. Ceci passe nécessairement par la reconnaissance par les autorités publiques de la langue basque et la légitimation de son enseignement et de son usage effectifs dans la vie publique du Pays Basque.

Certains auteurs de la fin du $19^{\circ}$ siècle, tel P. Broca ou encore M. Unamuno outreBidassoa, prédisaient, et souhaitaient même pour le bien des populations, la fin prochaine de la langue basque irrémédiablement condamnée, pensaient-ils, par le progrès des mœurs et des sociétés". C'était le temps où l'idée d'évolution était associée, par une extension de la théorie darwiniste hors du domaine de la biologie, à des classifications hiérarchisées des populations, des civilisations, et des langues dont le fondement réel résidait dans les préjugés racistes et nationalistes bien connus, y compris parfois chez des esprits se voulant éclairés. Ces conceptions n'ont plus cours, du moins dans le monde scientifique, et aucun linguiste ne songerait aujourd'hui à classer les langues selon leur degré d'évolution, de développement ou de dégénérescence. à partir de données typologiques, comme le proposait Schleicher ${ }^{12}$.

Nul n'oserait non plus défendre de bonne foi l'idée qu' un Pays Basque sans langue basque serait, du fait de la perte de sa langue, plus ouvert à la modernité et à l'universalité. Les faits ont démontré combien était fausse la croyance que la bascophonie pouvait être un obstacle à l'acquisition de langues plus répandues, à l'accès aux connaissances, et simplement à l'exercice de la pensée, comme avait pu le penser. par exemple, J. Vinson, dont le témoignage est d'autant plus significatif, que sa contribution et son attachement aux études basques ne sauraient être mésestimés. Prédisant la disparition prochaine de la langue basque, il formulait ce jugement bien illustratif $d$ " un certain type de pensée, qui a longtemps été, et demeure encore, sous-jacent à bien des comportements discriminatoires dans la définition des politiques linguistiques : "Al" point de vue social et humanitaire. il faut sans contredit se féliciter de la mort prochaine d'un idiome défectueux et incommode. qui est un obstacle redoutable à l'éducation des populations intelligentes. "(Vinson $1876: 14$ ).

A vrai dire, la crispation anti-moderniste et la fermeture à l'ouverture et à la diversité culturelle sont probablement plus aujourd'hui dans les Etats-Nations centralisés une caractéristique des opposants à la reconnaissance des langues autochtones et minorées. qu un trait des mouvements les défendant. Ceux-ci voient au contraire dans la pluralité linguistique, tant sociale qu'individuelle, non seulement une obligation. mais une chance d'enrichissement et d"épanouissement. 
Il est difficile d'évaluer quels seront à moyen terme les effets des efforts observés ces dernières années en faveur de la langue basque en particulier dans le domaine scolaire, hélas souvent à l'extérieur, ou en marge, du cadre institutionnel normal. Du moins peut-on espérer que la prochaine enquête sociolinguistique, probablement au début du prochain millénaire, laissera apparaître de manière plus nette les premiers effets des efforts entrepris.

Les résultats obtenus dans la Communauté Autonome, et même en Navarre, montrent de façon incontestable qu'il est possible de modifier, voire d'inverser, les tendances lourdes dans l'évolution des données sociolinguistiques dans des situations relativement comparables (en ce qui concerne les pourcentages des locuteurs) à celle exposée ici. Ainsi apparaît-il qu'une action volontaire dans le domaine de l'aménagement linguistique pourrait améliorer grandement une situation très préoccupante pour qui ne se résigne pas à voir ce témoignage exceptionnel de l'Europe linguistique que représente la langue basque progressivement éliminé de la vie sociale de cette partie du Pays Basque.

\section{Annexe}

Afin de permettre au lecteur de situer les chiffres relatifs au Pays Basque aquitain parmi ceux concernant l'ensemble du Pays Basque, nous offrons dans cette annexe un tableau général en chiffres absolus et pourcentages, avec un regroupement par provinces (sondage 1996 sur l'ensemble du Pays Basque, répartition des résultats sur la population d'après les recensements de 1990 pour le Pays Basque Nord et 1991 pour le Pays Basque Sud).

Pourcentage el nombre des hascophones el non bascophones agés de 16 ans ou plus dans l'ensemble du Pays Basque el dans les differcnles provinces (Soule et Basse-Navarrc regroupees)

\begin{tabular}{|c|c|c|c|c|}
\hline & $\begin{array}{l}\text { bascophones } \\
\text { actifs }\end{array}$ & $\begin{array}{l}\text { bascophones } \\
\text { passifs }\end{array}$ & $\begin{array}{c}\text { non } \\
\text { hascophones }\end{array}$ & Total \\
\hline \multirow[t]{2}{*}{ Alava } & 8200 & 34100 & 181700 & 234000 \\
\hline & $7.79 \%$ & $14.57 \%$ & $77.64 \%$ & $100 \%$ \\
\hline \multirow[t]{2}{*}{ Biscaye } & 175800 & 179100 & 61700 & 971900 \\
\hline & $18.10 \%$ & $18.42 \%$ & $63.48 \%$ & $100 \%$ \\
\hline \multirow[t]{2}{*}{ Guipuscoa } & 254200 & 77100 & 364800 & 452700 \\
\hline & $44.39 \%$ & $13.46 \%$ & $42.15 \%$ & $100 \%$ \\
\hline \multirow[t]{2}{*}{ Navarre } & 43200 & 44700 & 364800 & 452700 \\
\hline & $9.54 \%$ & $9.88 \approx$ & $71,33 \%$ & $100 \%$ \\
\hline Labourd & 35900 & 15900 & 128900 & 180700 \\
\hline (avec Bayonne + Buucau) & $18.85 \%$ & $8.82 \%$ & $71.33 \%$ & $100 \%$ \\
\hline \multirow[t]{2}{*}{ Soule \& Basse Navarre } & $20 \mathrm{sin})$ & 3900 & 7500 & 31700 \\
\hline & $63.99 \%$ & $12.27 \%$ & $23.74 \%$ & $100 \%$ \\
\hline \multirow[t]{2}{*}{ TOTAL Pays Basque } & $5476(x)$ & 354800 & 1541300 & 2443600 \\
\hline & 22.412 & $1452 \%$ & $63.07 \%$ & $100 \%$ \\
\hline
\end{tabular}




\section{Bibliographie}

Aizpurua. X. 1995. La continuité de la langue basque, Eusko Jaurlaritzaren Argitalpen Zerbitzu Nagusia, Vitoria-Gasteiz.

Bonapare, L-L. 1869. Carte des sept provinces basques montrant la délimitation actuelle de l'euscara et sa division en dialectes et variétés (1863), Londres. Paris.

Broca, P. 1875. Sur l'origine et la répartition de la langue basque, Emest Leroux.

Brunot, F. 1927. Histoire de la langue française des origines à nos jours, Tome IX : La Révolution et l'Empire, 2ème édition, 1967, Colin, Paris.

Bureau des Longitudes. 1809. Annuaire présenté à S. M. I'Empereur et Roi parle Bureau des longitudes, Imprimerie Nationale, Paris.

HPIN (Hizkuntza Politikarako Idazkaritza Nagusia). 1989. Soziolinguistikazko mapa. 1986. urteko erroldaren araberako euskal autonomi elkarteko azterketa demolinguistikoa, Eusko Jaurlarizaren Argitalpen Zerbitzu Nagusia, Vitoria-Gasteiz.

Granja, J. J. 1986. 'Julien Vinson, el euskera y un polémica del XIX', ASJU 20-I, 217-235.

Malberg. B. 1991. Histoire de la linguistique. De Sumer à Saussure. PUF, Paris.

Michel, F. 1857. Le Pavs Basque, sa population, sa langue, ses mours et sa musique, édition fac-similée, 1983, Elkar, Bayonne et Saint-Sébastien.

Oyharçabal, B. 1992. 'Euskararen mugez egin lehen mapak (1806-1807)', Euskaltzaindia, Iker 6, Bilbo, 349-366.

Oyharçabal. B. 1994. 'Les documents recueillis lors des enquêtes linguistiques en Pays Basque durant la période révolutionnaire et le Premier Empire', in J.B. Orpustan (édit.) La Révolution française dans l' histoire et la littérature basques du XIX' siècle. éditions Izpegi, Baigorri, 63-119.

Reclus, E. 1867. 'Les Basques, un peuple qui s'en va', in Revue des Deux Mondes, 15 mars 1867.

Unamuno, M. de - 1884. Crítica del prohlema sohre el origen y prehistoria de la raza vasca, thèse de doctorat (Facultad de Filosofia y Letras de Madrid) publiée in La raza y el vascuenze - En torno a la lengua española, col. Austral, Espasa-Calpe, Madrid.

Velasco, L. 1879. Los euscaros en Alava, Guipuzcoa y Vizcava. Sus origenes, historia, lengua, leves, costumbres y tradiciones, Oliveres, Barcelone.

Vinson, J. 1876. Le basque et les langues américaines, Communication au Congrés intemational des américanistes (1875, Nancy), Maisonneuve, Paris.

Vinson, J. 1878. 'Revista euskara', in Revue de Linguistique et Philologie comparée. 09, 466-467.

Vinson, J. 1882. Les Basques et les Pays Basque, Léopold Cerf, Paris.

Vinson, J. 1891-1897. Essai d'une bibliographie de la langue basque, réédition fac-similée avec les commentaires de J. Urquijo et une préface de L. Michelena dans les suppléments de ASJU, n9, 1984. Saint-Sébastien.

Vinson. J. 1900. 'La tradition au Pays Basque', in Revue de Linguistique et Philologie comparée, 33.

Yrizar, Pedro de --. 1973. Los dialectos y variedades de la Lengua Vasca. Estudio linguistico-dlemografico, publié comme publication séparée par le Boletin de la Real Sociedad de los Amigos del Pais. 29, 1-3. 


\section{NOTE}

1. Texte d'une communication présentée lors du colloquc sur les langues régionales de France tenu à Philadelphic du 4 au 6 octobre 1996 au Penn Language Center (University of Pennsylvania).

2. F. Michel (1857:2-3) apponte les indications suivantes sans preciser la manière dont il lcs a recucillics : Le Labourd compte environ soixante mille Basques (...). la Soule environ trente mille (...). et la basse-Navarre quarante cing mille. La somme est donc de 135000 . E. Reclus (1867:2) indique pour sa part avoir cffectue dans la zonc bascophone un relevé de la population commune par commune pour 1866 et obtcnu le nombre de 123810 , qu'il restreignait néanmoins un peu, car ajoutait-il ; mais il faut défalquer les útrangers domiciliés dans les villes de Saint Palais. Mauléon. Saint Jean de Luz etc. Le décomple de L-L. Bonapartc est rapporte dans Vinson (1876:16). Vinson (1882:68). quant à lui, après avoir interpréte les resultats du recensement de $1876 \mathrm{cn}$ fonction de la cartc de Bonaparte, propose une cstimation identique avcc la partition suivante : 65000 dans l'arrondissement de Bayonnc (sur 102000 habitants), el 60000 dans l'arrondissement de Mauléon (sur 63000 habitants). Je ne mentionne pas dans le texte le dénombrement de 80000 bascophoncs proposé par Velasco (1879:488), manifestement mal informé.

Ces estimations ne concerncnt que la zone bascophonc. mais il va sans dire que dans la zone contiguc également. particulièrement dans l'agglomeration bayonnaise, on rencontrait également des bascophoncs. C"est ce qu' indiquait le Préfet Castellane dans sa lettre de 1806 au Ministre de l'Interieur : Je n' ai pas besoin de faire remarquer à volre excellence que l'exrême voisinage ou de rapports d'intétêts font que dan. heaucoup de communes de la langue française une grande partie d' habitants parlent le basque. C'est li un effer naturel des relations entre ces peuples voisins et qu on aperçoit dans toutes les frontières. (cf. Oyharçabal $1994: 80$ ).

3. Jc rappelle ici les principaux resulats de celte enquétc pour le Pays Basque Nord (cf. Aizpurua 1995 61):

$\begin{array}{lrr}\text { - hascophoncs actifs : } & 69100 & 33 \% \\ \text { - bascophonc passifs : } & 14700 & 07 \% \\ \text { - non bascophoncs : } & 125100 & 60 \% \\ \text { - population totalc: } & 208000 & 100 \%\end{array}$

Sclon l'enquêtc de 1996, la proportion de bascophones actifs esı nettement inférieure (26\%). Bien sûr. on ne saurait attribuer cet ćcart a la seule baisse effective, dans une telle proportion durant une période aussi brève. du nombre des locutcurs. mais surtout aux conditions de réalisation des enquêtes.

4. Nous ne mentionnerons pas ici ics résultats du questionnaire concernant les opinions et les compurtements. qui constitucnt néanmoins une partie importante de l'enquête.

5. Pour la partic aquitainc c'cst l'NSEE qui a procéde à la sćlcction de l'échantillon.

6. L'appcllation de zonc bascophone traditionnelle fait référence à la zonc bascophone tclle qu elle fut dćlimitéc au sičle demicr par le Prince Bonaparte. en 1863 , el qui ne differc guère d'ailleurs de cclic du Préfct Castellanc réaliséc en 1806. Le principal ccart par rappont au Pays Basque en quelque sorte sociogéographique ou administratif tel qu'il cst défini dans l'enquête de 1996 concerne les cantons de Biarrilz.. Anglet. Bayonne et du Boucau, qui n'étaient pas inclus dans la zone bascophonc délimitce avec précision par Bonaparte.

7. L'cnquêtc ayant cu licu durant l'hiver, on peut considerer neanmoins que pour les personnes clfec. tivement interrogces il s'agissait de la résidence principalc.

8. Comme ce sunt les personnes enquêtees qui definissent leur propre compétence, le risque de distorsinns ou d'insincérité des réponses cxistc. On a ainsi parfois constaté dans d'autres contrées unc sur-évaluation de la compétence dans la langue autochtonc dans les cnquêtes démolinguistiques. Toutefois. Jans le cas préscnt. la procédure de réalisation des enquêtes a permis de réduire au maximum cet ćcucil. En premicr licu toutcs les cnquĉtes ont eu licu après avertissement par courrier de la sollicitation et exposition de son objet. cl cnsuitc prise de rendez-vous par téléphone avec la personnc qui, au sein du foyer sélectionné. devait répondre au questionnaire (choix fixé de manièrc alcatoire). En second lieu. les enquêtcurs posaicnt unc question préalable visant à déterminer si la personne possédait bien ou asscz bien le basque. $\mathrm{CL}$ cn cas de réponsc positive, utilisaicnt le basque comme languc de communication au cours de l'enquêle (Uans lc respect néanmoins des options linguistiques des personnes interrogées. si celles-ci refusaicnt l'cmploi du basque ou preferaicnt emplnyer lc francais ou l inversc). Enfin. la richesse du questionnaire permettant de nombrcux recoupements au cours d un cntreticn relativement long (environ trois-quarts d'heure pour les bascophones). favorisait des réponses. non sculcment sincères. mais aussi mesurees ct séricuses. Commc indiqué. les échantillons des foyers sollicités pour l'cnquête ont êté fournis par l'INSEE. et les enquêtcurs. tous billingues. ont reçu. sous l'ćgide de l'Institut Culturel Basque. unc formation préalable. de la par de 
I'INSEE. Les précautions priscs dans la répartition des ćchantillons. comme les conditions de réalisation đes cnquêtcs. constitucnt les principales améliorations apportées par rapport à l'enquête de 1991.

9. Lc faiblc nombre de bascophoncs parmi les personncs interrogées interdit en pratique l'analyse statistique des sous-groupes que l'on pourrait distinguer au sein de cette population.

10. Les responsabics de l'INSEE ayant collaboré a la réalisation de l'enquête recommandent le regroupement des zones 2 et $2^{\circ} \mathrm{cn}$ une zone unique pour l'exploitation des données, de manière à atteindre un Échantillon atteignant un seuil de $\mathbf{4 0 0}$ environ. niveau minimum souhaitablc pour l'exploitation. Ils indiquent toutcfois que pour la seulc quantification des groupes de locuteurs, la partition n' altère pas les résultals de manière significalive. Ceci est confirmé par l'examen des résultals obtenus après regroupement des echantillons, très proches de ceux obtenus par simple addition des résultals dans chacune des zones:

Zonc 2 unique (Labourd sans l'agglomération bayonnaise) :

$\begin{array}{llr}\text { bascophones actifs : } & 27.499 & 31 \% \\ \text { bascophoncs passifs : } & 12352 & 14 \% \\ \text { non-bascophoncs : } & 48753 & 55 \% \\ \text { Total : } & 88604 & 100 \%\end{array}$

11. Liexpression la plus claire de ce sentiment parmi les bascologucs se retrouve chez J. Virnson, lequel, à propos de la déclaration d'intention de l'association navarraise Euskara. créé en 1878. de s'opposet a la disparition de la languc et de promouvoir une litteraturc d'expression basque, déclarait : "c est précisément parce que cer idiome est incompatible avec leur civilisation actuelle - loute espagnole - qu'il ne peut plus vivre et qu 'il doit fatalement disparaître. Le basque n' est ni une langue litteraire ni une langue convenable aux instincts dêmocratiques de notre siècle" : (Vinson 1878 : 466-7). Sur le fondement scientiste sous-jacent à celte altitude. voir la notc 12.

12. A. Schleicher (1821-1868) fut l'une des figures de la linguistique historique du milieu du lycme siecclc. Quatrc années après la publication des thèses de Darwin sur l'origine des especes et la sélection naturellc (1859), il publait un ouvrage sur l'application de la thcoric darwinienne a la linguistique, ainsi que le remarquc B. Malberg (1991: 302). C'est à Schlcicher que l'on doit la célebbre classification des langues selon lcur typologic morphologiquc (langues isolantes. langues agglutinantcs et langues flexionnelles), et qu'il faisait correspondre pour sa part à des stades d'évolution des langues. Dans le monde de la bascologic. Ies idécs de Schicicher furent surtout pontés par J. Vinson. qui ne cachait pas sa dette à son égard (1876:9). Cela le conduisit d'ailleurs. en cenaines circonstances, face à des zélateurs parfois ridiculement enthousiastes du hasquc. à formuler des jugements péchant par excès inverse : "ce remarquable reste des vicux ages [il s'agit évidemment du basquc] correspond manifestement à une mentalité inférieure et d̀ une période primitive de l'humanite." (Vinson 1900 : 292-3). (La mise en relief du passage en gras n'appartient pas à loriginal).

13. Pour unc ćtudc concemant les idées de Vinson concemant le basque. on se reportera notamment à Granja (1986). Les idécs linguistiques de Vinson sont également situécs dans le contexte général de la linguistique el des ćludes hasques de la fin du $19^{*}$ siecle par Michelena dans sa préface à la réédition de la bibliographic hasque de Vinson (1891-1897). 